\title{
Editorial
}

\section{Swarm Intelligence in Engineering 2014}

\author{
Baozhen Yao, ${ }^{1}$ Fang Zong, ${ }^{2}$ Bin $\mathrm{Yu}^{3}{ }^{3}$ and Rui $\mathrm{Mu}^{4}$ \\ ${ }^{1}$ School of Automotive Engineering, Dalian University of Technology, Dalian 116024, China \\ ${ }^{2}$ College of Transportation, Jilin University, Changchun 130022, China \\ ${ }^{3}$ Transportation Management College, Dalian Maritime University, Dalian 116026, China \\ ${ }^{4}$ Delft University of Technology, NL-2628 BX Delft, Netherlands
}

Correspondence should be addressed to Bin Yu; ybzhyb@163.com

Received 19 November 2014; Accepted 19 November 2014

Copyright (C) 2015 Baozhen Yao et al. This is an open access article distributed under the Creative Commons Attribution License, which permits unrestricted use, distribution, and reproduction in any medium, provided the original work is properly cited.

Swarm intelligence (SI) is an artificial intelligence technique based on the study of cooperation behaviors of simple individuals (e.g., ant colonies, bird flocking, animal herding, and bees gathering honey) in various decentralized systems. The population, which consists of simple individuals, can usually solve complex tasks in nature by individuals interacting locally with one another and with their environments. Although a simple individual's behavior is primarily characterized by autonomy, distributed functioning, and selforganizing capacities, local interactions among the individuals often lead to a global optimal solution. Therefore, swarm intelligence is a promising way to develop powerful solution methods for optimization problems in engineering.

However, for large and complex engineering problems, SI algorithms often consume too much computation time due to the stochastic features of their searching approaches. For this reason, there is a potential requirement to develop efficient algorithms that are able to find solutions under limited resources, time, and money in real-world applications.

This special issue is aimed at highlighting the most significant recent developments on the topics of SI and to apply SI algorithms in real-life scenarios. Papers selected for this special issue present new findings and insights into this field. A broad range of topics are discussed, especially in the following areas: benchmarking and evaluation of new SI algorithms, convergence proof for SI algorithms, comparative theoretical and empirical studies on SI algorithms, and SI algorithms for real-world application.

Some works focus on the application of SI in the area of traffic and transportation. For example, in the paper entitled "Road network vulnerability analysis based on improved ant colony algorithm," Y. Wang et al. developed a novel approach based on an improved ant colony algorithm (IACA) to evaluate the vulnerability as well as identify the critical infrastructures of a real-world road network. They proved the high computational efficiency of the IACA-based approach when applied in large-scale networks and presented the dependence of road network vulnerability on the ration between traffic demand and road capacity.

In the paper titled "Hybrid model for early onset prediction of driver fatigue with observable cues," M. Zhang et al. presented a hybrid model for the early onset prediction of driver fatigue. The hybrid model consists of three submodels which separately solve the three stages of the prediction problem. The support vector machine (SVM) is incorporated in the hybrid model for predicting the early onset driver fatigue state, while the genetic algorithm is used for optimizing parameters in the SVM. The approach has a good performance in the numerical test for the early onset prediction of driver fatigue.

In the paper titled "Real-time arterial coordination control based on dynamic intersection turning fractions estimation using genetic algorithm," P. Jiao et al. established a realtime arterial coordination control model based on dynamic intersection turning fractions estimation. They developed an improved genetic algorithm to solve the dynamic intersection turning fractions estimation model, the solution of which was the inputs of the real-time arterial coordination control model. To solve the real-time arterial coordination control model, the authors proposed another improved genetic algorithm. Simulation experiment based on actual data proved the superiority of their approach. 
In the paper entitled "Study of on-ramp PI controller based on dural group QPSO with different well centers algorithm," T. $\mathrm{Wu}$ et al. proposed a new quantum behaved particle swarm optimization (QPSO) algorithm, which is the dual-group QPSO with different well centers (DWC-QOSO) algorithm. This novel algorithm could slow down the disappearance of population diversity and thus improve the global searching ability. The successful application of the DWC-QOSO algorithm in the on-ramp traffic control simulation indicated its powerful potential in the study of on-ramp PI controller.

In the paper entitled "Decision of multimodal transportation scheme based on swarm intelligence," $\mathrm{K}$. Lei et al. considered three elements of transportation costs, time, and risks when establishing a multimodal transportation scheme decision model. To solve the model, they proposed an improved SI algorithm combining particle swarm optimization (PSO) and ant colony (AC) algorithm. The numerical test showed that their algorithm inherited the advantages of both PSO and AC and had good ability to solve the decision problem of multimodal transportation scheme.

Also, the SI is discussed in the area of computer science. In the paper entitled "Multipeak mean based optimized histogram modification framework using swarm intelligence for image contrast enhancement," P. Babu et al. proposed a PSO based approach to enhance the contrast and preserve essential details for any given image. They divided the histogram of an image into two subhistograms. The two subhistograms can be modified by optimal enhancement parameters which are found by the particle swarm optimization algorithm.

In the paper entitled "Binary structuring elements decomposition based on an improved recursive dilation-union model and RSAPSO method," Y. Zhang et al. developed an improved dilation-union model for decomposing the structuring elements of any given shape, and they used the restarted simulated annealing particle swarm optimization as the algorithm of their model. Experiments showed that the proposed method outperformed most existing methods.

In the paper entitled "Applications of PCA and SVM-PSO based real-time face recognition system," M.-Y. Shieh et al. used the support vector machine (SVM) which could be an optimal classifier of image recognition system to improve the validity of real-time face recognition systems. The particle swarm optimization is used by the authors to implement a feature selection, results of which are then input to the SVM for calculating the fitness value of classification.

There are also some works focusing on the application of SI in the operation research and management science. In the paper entitled "Core business selection based on ant colony clustering algorithm," Y. Lan et al. proposed a method based on the ant colony clustering algorithm to identify the core business of an enterprise. By applying the method, the authors successfully found the core business of Tianjin Port from its ten main businesses.

In the paper entitled "A framing link based tabu search algorithm for large-scale multidepot vehicle routing problems," $\mathrm{X}$. Zhang et al. presented a framing link based Tabu search algorithm for solving a large-scale multidepot vehicle routing problem (LSMDVRP). Framing links are extracted from continuous great optimization of current solutions and updated iteratively in the whole algorithm. The proposed algorithm was tested with 18 LSMDVRP instances and the results indicated that it had high computation speed and reliability.

In the paper entitled "Forecasting dry bulk freight index with improved SVM," Q. Han et al. attempted to forecast the short-term trend of dry bulk freight index with an improved SVM model which incorporated the wavelet transform. As the dry bulk freight index is usually influenced by random events in the freight market, the wavelet transform is then combined to the SVM to denoise the index data series. The genetic algorithm is used to optimize parameters of the improved SVM. The prediction method displayed high accuracy in the experiment based on real data.

In the paper entitled "Artificial intelligence mechanisms on interactive modified simplex method with desirability function for optimising surface lapping process," the authors applied the harmony search and firefly algorithms to optimize the influential parameters of surface lapping process of disk clamps.

In the paper entitled "An adapted firefly algorithm for product development project scheduling with fuzzy activity duration," M. Huang et al. developed an adapted fuzzy firefly algorithm to solve a mathematical model which was built for scheduling new product development project with uncertain activity duration. The effectiveness and efficiency of the proposed algorithm were validated with benchmark experiments as well as a real-world example.

Besides those mentioned above, some authors present the application of SI in the electrical and electronic engineering. In the paper entitled "Short-term power generation energy forecasting model for small hydropower stations using GASVM," G. Li et al. used a GA-based SVM model to predict short-term power generation energy of small hydropower and the numerical test proved the better performance of the GA-SVM forecasting model compared with the ARMA model.

In the paper entitled "Short-term wind speed forecasting using support vector regression optimized by cuckoo optimization algorithm," J. Wang et al. proposed a hybrid forecasting model combining recurrence plot (RP) with support vector regression (SVR) to predict short-term wind speed series. In the hybrid model, the RP is used to analyze the wind speed series and select input variables for the SVR which is employed for short-term prediction, while the parameters of the SVR are optimized by three SI algorithms, respectively, say the GA, the PSO, and the cuckoo optimization algorithm (COA). The experimental results indicated that the forecasting model based on COA-SVR outperformed the other two models, especially in the context of jump samplings and multistep prediction.

In the paper entitled "Swarm intelligence-based smart energy allocation strategy for charging stations of plug-in hybrid electric vehicles," I. Rahman et al. used the gravitational search algorithm (GSA) and the PSO to optimize the charging allocation strategy for extensive participation of plug-in hybrid electric vehicles, respectively, and analyzed the advantages as well as disadvantages of the two SI-based methods in the numerical experiment. 
In the paper entitled "Swarm intelligence-based hybrid models for short-term power load prediction," J. Wang et al. combined the singular spectrum analysis (SSA) with the seasonal autoregressive integrated moving average (SARIMA) and the SVR, respectively, and developed two improved forecasting models, that is, the SSA-SARIMA and the SSASVR, to implement short-term power load prediction. In order to optimize the parameters of the two hybrid models, the cuckoo search is employed. The experimental results showed that the application of CS could further improve the prediction accuracies of both the two models.

In the paper entitled "A swarm random walk based method for the standard cell placement problem," the authors presented a new SI-based method, the swarm random walk method, to optimize the standard cell placement on large-size chip. According to the resulting placement in the benchmark experiment, the proposed method could be very competitive for solving the standard cell placement problem.

In the paper entitled "Pattern synthesis of planar nonuniform circular antenna arrays using a chaotic adaptive invasive weed optimization algorithm," to optimize the nonuniform circular antenna arrays, $\mathrm{H}$. Wu et al. revised the invasive weed optimization (IWO) by combining the chaotic search method into the IWO with adaptive dispersion and obtained a novel IWO called chaotic adaptive invasive weed optimization (CAIWO) which has better convergence speed as well as global searching ability.

There are several papers that apply SI in the civil engineering. In the paper entitled "Displacement prediction of tunnel surrounding rock: a comparison of support vector machine and artificial neural network," Q. Wu et al. used two methods, that is, the SVM and artificial neural network (ANN), to separately predict tunnel surrounding rock displacement, and they found that the SVM-based method was more robust and accurate than the ANN-based method for the displacement prediction of tunnel surrounding rock.

In the paper entitled "Damage identification of bridge based on modal flexibility and neural network improved by particle swarm optimization," H. Liu et al. developed a twostage method to identify damage of bridge. In the first stage, modal flexibility indices are employed to localize damage and then an ANN whose parameters are optimized by PSO is used in the second stage to identify damage severity. The numerical simulation proved the feasibility and superiority of the proposed method.

In the paper entitled "Optimal sensor placement for latticed shell structure based on an improved particle swarm optimization algorithm," X. Zhang et al. proposed a new improved particle swarm optimization (IPSO) algorithm to optimize the sensor placement in large-scale structures for the structural health monitoring. In the case study, they proved the feasibility of the IPSO-based method, as well as its superiority to other PSO algorithms in terms of convergence speed and precision.

Communication is another realm where SI can be applied. In the paper entitled "Optimal sizing of a photovoltaichydrogen power system for HALE aircraft by means of particle swarm optimization," V. Sanchez et al. attempted to design a power supply system for high altitude long endurance
(HALE) aircrafts which could provide a wide range of telecommunication services. The main problem they met resided in the size optimization for the system, and they used the PSO to solve the optimal sizing problem. Case study showed that the power supply systems calculated by PSO could work efficiently and steadily for long time flights.

In the paper entitled "Location prediction-based data dissemination using swarm intelligence in opportunistic cognitive networks," J. Li et al. applied the ant colony optimization to address the data dissemination problem in the opportunistic cognitive networks. Real-world simulation indicated that the routing scheme for conveying message optimized by the ant colony algorithm had better performances.

There are also two papers discussing the SI in automotive engineering. In the paper entitled "Research of ant colony optimized adaptive control strategy for hybrid electric vehicle," L. Li et al. develop an ant colony optimization based method to dynamically determine energy management control strategy of hybrid electric vehicles (HEVs) in different driving cycles. Based on a certain type of driving cycle identified by a fuzzy driving cycle recognition algorithm, the ant colony optimization algorithm is used to optimize the control parameters in the corresponding context. The optimization method is validated in the simulation experiments.

In the paper entitled "Research of obstacle recognition technology in cross-country environment for unmanned ground vehicle," Z. Yibing et al. presented a multistep approach incorporating filtering algorithm and multifeature fusion algorithm based on Bayes classification theory to solve the obstacle recognition problem of unmanned ground vehicles in cross-country environment. The robustness and accuracy of the algorithm are well proved in the simulation test.

Some works use the SI in robotics. In the paper entitled "PSO-based robot path planning for multisurvivor rescue in limited survival time," N. Geng et al. studied the optimal rescue path planning of a robot when using it in urgent and dangerous circumstances. To solve the problem, the authors developed a novel improved PSO incorporating particle updating, insertion and inversion operators, and a rapid local search method. The simulation results presented the feasibility and efficiency of the algorithm in finding optimal paths.

The robot path planning problem is also attempted by J. J. Liang et al. In their paper entitled "Comparison of three different curves used in path planning problems based on particle swarm optimizer," they employed the dynamic multiswarm particle swarm optimizer to search the optimal parameters of three commonly used curves which were usually applied in robot path planning problems.

The following four papers mainly focus on enhancing the representative SI algorithms. In the paper entitled "Hybrid biogeography based optimization for constrained numerical and engineering optimization," Z. Mi et al. proposed a new hybrid biogeography based optimization (HBBO) algorithm which contained chaotic search and a new mutation operator combining differential evolution mutation operator with simulated binary crosser of genetic algorithm. To achieve global optimal, the differential evolution mutation operator is still used in the $\mathrm{HBBO}$ to provide an update on half 
population. The HBBO was tested with 12 benchmark and four engineering optimization problems, and results showed that the HBBO outperformed other evolutionary algorithms, especially for constrained optimization problems.

In the paper entitled "A modified artificial bee colony algorithm based on search space division and disruptive selection strategy," Z. He et al. improved the artificial bee colony (ABC) algorithm from three aspects. They proposed a new initialization method to generate high quality initial solutions, a disruptive selection strategy to enhance population diversity, and a novel definition of the scout bee phase.

In the paper entitled "Quantum behaved particle swarm optimization algorithm based on artificial fish swarm," the authors introduced adaptive parameters and swarm and follow activities to the existing quantum behaved particle swarm optimization (QPSO) algorithm and obtained a new QPSO called quantum particle swarm optimization algorithm based on artificial fish swarm, which was validated to have better optimization ability than QPSO.

In the paper entitled "The inertia weight updating strategies in particle swarm optimisation based on the beta distribution," the authors compared the performances of different PSOs under different selected random updating strategies of inertia weight. By using 28 benchmark functions, they found that the multiswarm PSO combining two updating strategies of inertia weight was the best.

\section{Acknowledgments}

These articles present rich and valuable advancements that SI technologies have made for solving problems in engineering. We would like to thank all the authors for their excellent work and contributions to this special issue. We would also like to express our gratitude to all the reviewers for their fundamental work and patience in assisting us.

Baozhen Yao Fang Zong

Bin $\mathrm{Yu}$

Rui $M u$ 


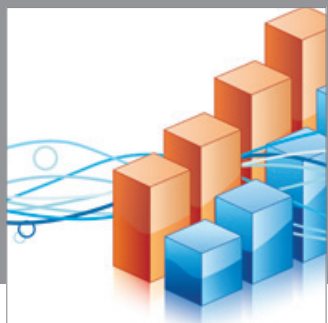

Advances in

Operations Research

mansans

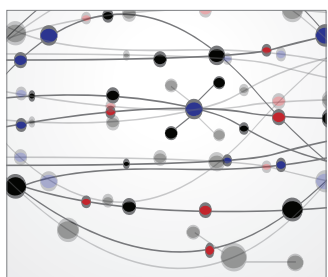

The Scientific World Journal
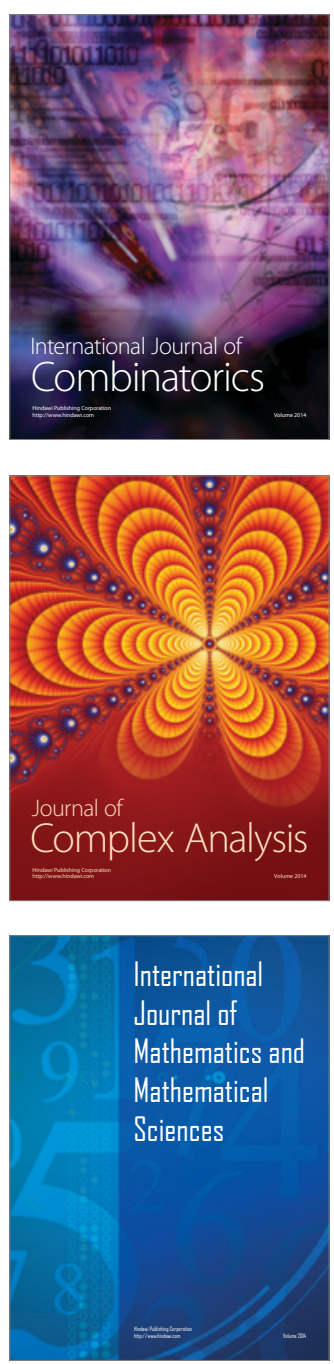
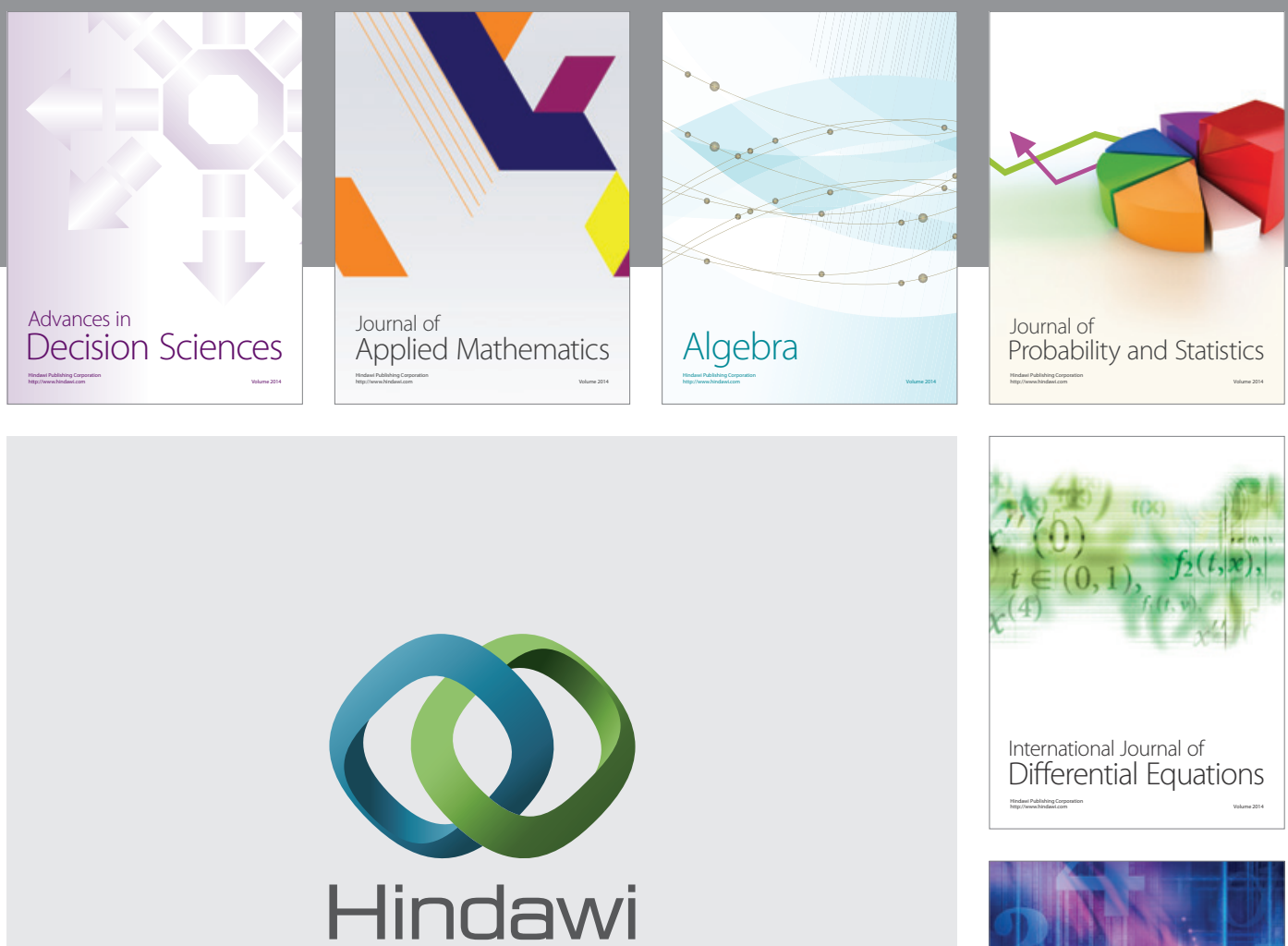

Submit your manuscripts at http://www.hindawi.com
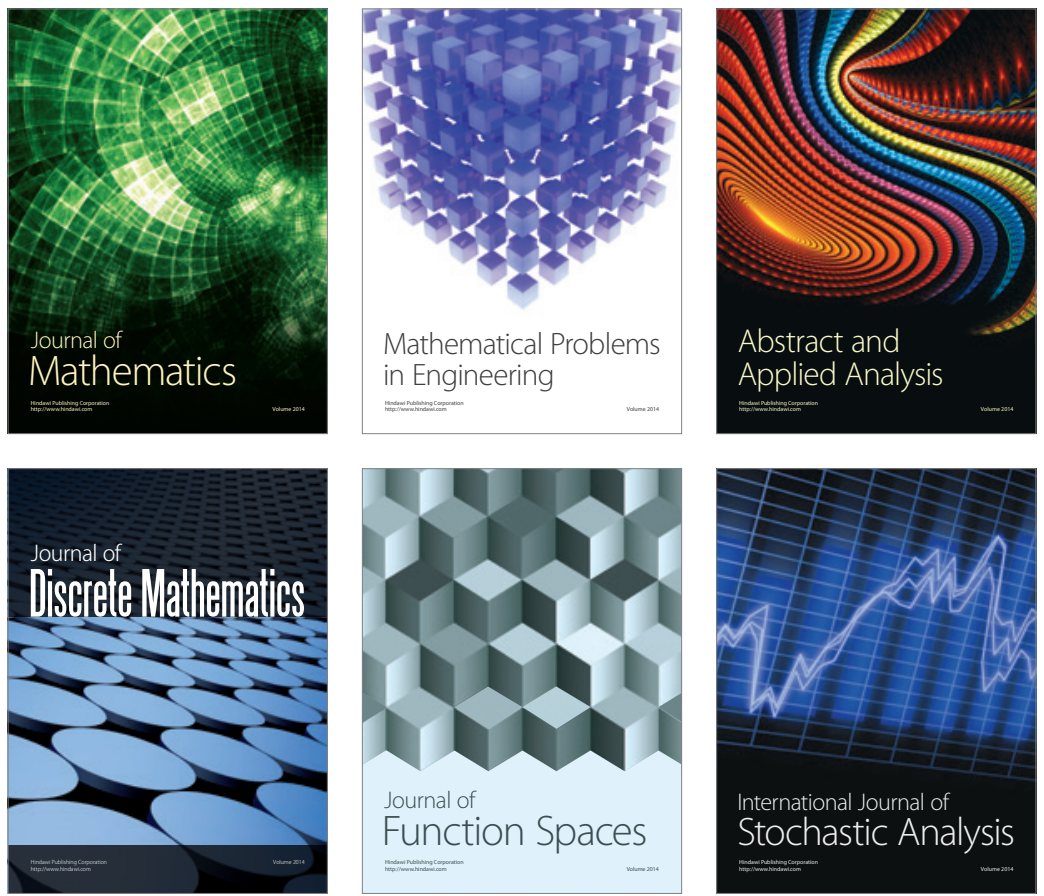

Journal of

Function Spaces

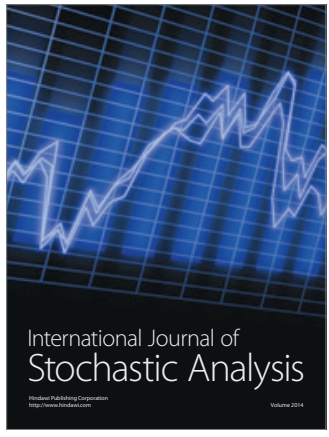

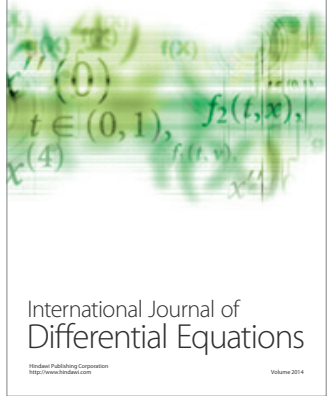
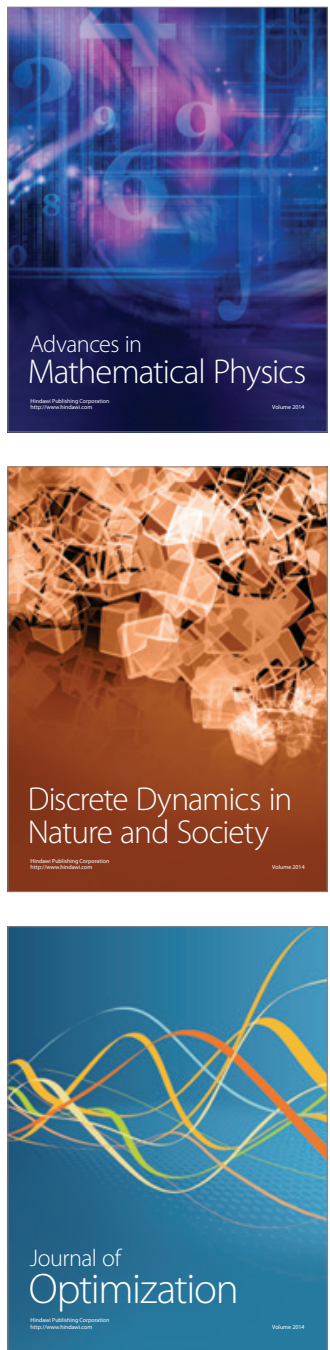